\title{
Un arbre, une forêt, un paysage. La contribution du Touring Club de France en faveur du reboisement
}

\author{
Pierre-Olaf Schut ${ }^{1}$, Matthieu Delalandre ${ }^{2}$ \\ 1 Histoire contemporaine, Université Paris-Est, EA 3350 Analyse comparée des pouvoirs, 77454 Marne-la-Vallée cedex 2, France \\ 2 Sociologie, Université Paris-Est, EA 3350 Analyse comparée des pouvoirs, 77454 Marne-la-Vallée cedex 2, France
}

Si les historiens académiques montrent un intérêt croissant pour les environmental studies, on constate symétriquement une recherche de profondeur historique de plus en plus fréquente chez les chercheurs qui s'interrogent sur le lien entre sciences, pratiques de la nature et représentations sociales dominantes. Pour NSS, cette prise en compte de la temporalité représente un défi méthodologique important dans l'éventail des pratiques interdisciplinaires, la temporalité n'étant évidemment pas l'apanage des seuls historiens. La prendre en compte exige toutefois de mobiliser l'un de leurs concepts-clés, celui de « contexte ", particulièrement difficile à cerner quand il s'agit, justement, de "nature ». C'est tout l'intérêt de l'article proposé par Pierre-Olaf Schut et Matthieu Delalandre, d'appliquer à l'action en faveur du reboisement du Touring Club au tournant des années 1900 une réflexion sur la genèse des "politiques de la nature » dans une phase historique où la notion d'environnement n'existe pas sous sa forme actuelle, mais s'impose néanmoins comme triple enjeu scientifique, économique et esthétique.

La Rédaction

\section{Mots-clés :} environnement ; gouvernance ; reboisement ; Touring Club de France ; arbre

\section{Keywords:}

environment;

governance;

reforestation; Touring

Club de France; tree

\begin{abstract}
Résumé - Le Touring Club de France (TCF) fut créé en 1890 avec pour ambition de développer le tourisme sous toutes ses formes, ce qui impliquait également de préserver les paysages. C'est ainsi qu'au tournant du $X X^{\mathrm{e}}$ siècle, le club s'engage dans un combat contre la déforestation et pour le reboisement. L'analyse d'un corpus écrit composé notamment des publications de l'association et des services des Eaux et Forêts permet de mettre en avant la façon dont le TCF s'empare du sujet. D'une part, il fustige les coupes rases ; d'autre part, les bonnes pratiques sont largement mises en avant par la diffusion d'un Manuel de l'arbre, mais aussi par des incitations financières à la plantation d'arbres. Le Touring parvient également, grâce à ses relais au Parlement, à faire évoluer la législation afin d'améliorer la protection des bois et forêts.
\end{abstract}

\begin{abstract}
Trees, forests, landscapes. The contribution of the Touring Club de France towards reforestation. The Touring Club de France (TCF) was founded in 1890, with the ambition to promote tourism in all its forms. Landscape conservation was also part of this aim. This led the TCF to get involved at the turn of the $20^{\text {th }}$ century in an anti-deforestation campaign and to support reforestation actions, particularly in mountain areas. The analysis of a body of publications by the TCF and the national French Waters and Forestry Service as well as of debates in Parliament and the media highlights the way the TCF undertook to strengthen the impact of its actions and arguments by setting aside its social purpose in favour of a more general interest. On the one hand, the TCF censured clear-cuttings and advocated for a reasonable exploitation of forests; on the other hand, the wide distribution of a Manuel of the tree in primary schools aimed at disseminating good management practices and financial incentives encouraged private individuals and municipalities to play a part in tree planting. By means of a well targeted propaganda and through its contacts at the French Parliament the TCF also succeeded in getting the law to evolve towards better protection of wood and forests.
\end{abstract}

Auteur correspondant : M. Delalandre, matthieu.delalandre@u-pem.fr 
Le XIX ${ }^{\mathrm{e}}$ siècle est marqué par un développement productif et économique très important, tant sur le plan industriel qu'agricole, mais aussi sur celui des loisirs dans les franges les plus aisées de la société (Corbin, 1995). C'est ainsi que naît, en 1890 , le Touring Club de France (TCF), association dont le but est de développer le tourisme sous toutes ses formes, en offrant des facilités d'accès aux adhérents, mais aussi en contribuant à la conservation de tout ce qui constitue l'intérêt pittoresque ou artistique des voyages. Les élites urbaines, en promouvant le tourisme, rencontrent ainsi les aspirations des notables locaux pour mettre en avant les sites remarquables de leur région (Cousin et Réau, 2009). Or, si le développement du tourisme constitue, tout comme les transformations industrielles et agricoles, un marqueur de la civilisation moderne, il se révèle parfois incompatible avec celle-ci quand les ressources convoitées au nom d'intérêts économiques et productifs se situent dans des lieux touristiques reconnus.

L'État français avait déjà légiféré en 1887 pour favoriser la protection du patrimoine bâti. Mais des associations, notamment le TCF et le Club alpin français, ouvrent à nouveau le débat au début des années 1900, ce qui débouche sur la promulgation d'une loi en faveur de la protection des sites et monuments (Gauchon, 2002). Outre le renforcement de la loi de 1887, ce texte introduit une nouveauté majeure : la possibilité de protéger des sites naturels, reconnus pour leur valeur paysagère. Dans la continuité de cette action, le TCF montre un intérêt particulier pour les arbres et les forêts. Il ne se contente pas d'en apprécier les qualités paysagères et d'en défendre la préservation, mais il s'engage dans un combat initié près de cinquante ans plus tôt, celui de la reforestation et de la lutte contre le déboisement.

C'est à partir de 1750 que l'arbre apparaît, dans le discours des élites sociales, comme un élément déterminant de la qualité de l'environnement : « on ignore encore quel rôle il peut jouer dans le maintien des sols, quel emploi il fait du gaz carbonique, mais nul ne doute quant à sa capacité d'enrichir la terre et d'assainir 1'atmosphère » précise Andrée Corvol (1987b, p. 68). Un consensus semble en outre se dégager au sein de ces mêmes élites, pour constater un processus de dégradation des montagnes par déboisement, surexploitation et érosion des sols notamment.

Ce discours trouve un écho dans le nombre important d'inondations, de crues et d'avalanches, attribué en grande partie à l'archaïsme des sociétés rurales (Morin, 2010 ; Granet-Abisset, 2012), et va s'intensifier tout au long du XIXe pour culminer entre 1880 et 1914. L'intérêt pour ces catastrophes est amplifié par l'essor de la presse, particulièrement important au XIX ${ }^{\mathrm{e}}$ siècle (Corvol, 1987a).

Dans ce contexte, la première loi sur la restauration des terrains de montagne, votée en 1860 marque, à la suite des terribles inondations de 1856, le début d'une volonté d'intervention pour remédier au déboisement. La forêt se voyant attribuer un rôle régulateur pour absorber et restituer les eaux de pluie, la reforestation apparaît comme un moyen de prévention des risques de crues torrentielles, d'inondations et d'avalanches, réunissant les points de vue techniques, économiques et esthétiques des élites du siècle.

Héritier de cette culture de gestion éclairée des territoires, le TCF a le souci de valoriser et donc de préserver le patrimoine touristique. Parmi les sites naturels à sauvegarder, les bois et forêts semblent particulièrement fragiles et menacés. Aussi, le TCF se mobilise autour de cette problématique, cherchant à infléchir la politique de préservation dirigée par l'État dans le sens de ses préoccupations. Le XIX ${ }^{e}$ siècle est en effet marqué par la constitution d'un véritable appareil administratif, un « État forestier » qui se renforce, s'institutionnalise et s'autonomise (Buttoud, 1983 ; Kalaora et Savoye, 1986). Cet appareil administratif se caractérise par son corporatisme. Celui-ci fonde sa légitimité sur la compétence technique des représentants des Eaux et Forêts, ainsi que sur sa capacité à défendre l'intérêt général au sein du service public (Kalaora et Savoye, 1986).

Les forestiers ne constituent pas toutefois un corps monolithique : deux conceptions de l'aménagement des terrains de montagne s'y opposent, dans une crise « doctrinale » qui apparaît au grand jour en 1877, au moment de l'étude d'un nouveau projet de loi sur la restauration des terrains de montagne. La première conception, dominante, est centralisatrice et étatiste. Ses tenants apparaissent critiques à l'égard de la loi de 1882, qui entame les prérogatives de l'administration forestière. La seconde position, minoritaire au XIX ${ }^{\mathrm{e}}$ siècle, est souvent qualifiée de «sociale » : elle entrevoit la question forestière sous l'angle de la recherche d'un équilibre entre les intérêts des populations et les impératifs de gestion des sols. Il s'agit donc, en étant attentif au sort des populations pastorales, d'essayer de concilier intérêt général et interêts particuliers, d'inciter au reboisement plus que de l'imposer (Kalaora et Savoye, 1986). La forêt est ainsi au cœur de problématiques économiques et sécuritaires qui incombent directement aux pouvoirs publics, et qui ont donné lieu à des positions divergentes.

Aussi, pour servir sa cause, le TCF s'inscrit volontairement dans un discours non seulement patrimonial mais également économique et environnemental, pour rassembler autour de lui tous les acteurs susceptibles d'être intéressés par les questions forestières et de l'aider dans sa démarche. En cherchant à mobiliser les pouvoirs publics, il s'éloigne de son objet social pour utiliser des arguments susceptibles de convaincre les politiciens. Joignant le geste à la parole, le club agit en faveur du reboisement en s'investissant directement dans une mission de service public. Ce type d'approche avait déjà 
été employé par le TCF pour aménager les itinéraires routiers ou nautiques, afin d'assurer la sécurité des voyageurs. La question du reboisement lui fait faire un pas de plus dans un engagement patriotique qui dépasse les intérêts directs des touristes, pour mieux les servir en retour.

Notre analyse vise à mettre en lumière la façon dont le TCF défend sa cause auprès des pouvoirs publics, des municipalités et de la population, précisément en la rapportant à des enjeux qui dépassent les intérêts particuliers des voyageurs adeptes de tourisme. Nous montrons dans un premier temps que la défense de l'attrait touristique des sites boisés passe par un engagement patriotique et patrimonial : il s'agit en effet de sauvegarder le patrimoine naturel national pour pallier l'insuffisance de l'action publique dans ce domaine. Dans un second temps, nous analysons comment l'élargissement du champ d'intervention du TCF amène ses dirigeants à se positionner sur le plan économique : la forêt est un patrimoine, mais aussi une ressource naturelle dont on tire parti pour des besoins divers, et il convient de trouver des moyens de l'exploiter de façon durable et pérenne. Sa sauvegarde peut en effet constituer une source de richesse économique. Enfin, nous mettons en lumière la façon dont cet argumentaire économique s'enrichit d'un discours et d'actions d'une portée encore plus générale relatifs à la préservation des montagnes et à la sécurité des populations, menacées par les risques de crues et d'inondations. S'appropriant une mission régalienne, l'association entend lutter contre la déforestation et favoriser le reboisement des terrains de montagne afin de diminuer ces risques.

Les sources de ce travail sont centrées sur le TCF. Elles s'appuient principalement sur sa revue mensuelle, ses publications et les archives du club. Comme les interactions avec les pouvoirs publics sont nombreuses, nous mobilisons des publications des services des Eaux et Forêts, les débats de la Chambre liés à notre objet et quelques extraits de la presse. Ce corpus est analysé qualitativement pour la période qui s'étend de 1905 à 1913, c'est-à-dire du début de l'engagement du TCF dans cette cause jusqu'à une forme d'aboutissement avec la promulgation d'une loi en 1913 visant à protéger les bois et forêts. L'étude du corpus montre que l'investissement du club s'opérationnalise de trois façons différentes et complémentaires. Premièrement, il produit des arguments, des discours reflétant ses propres conceptions, destinés en premier lieu aux membres eux-mêmes (à travers sa revue), mais aussi aux acteurs politiques. Deuxièmement, il agit, grâce à des relais locaux, au plus près des pouvoirs locaux et des populations. Troisièmement, il intervient à un niveau plus central en essayant de peser sur la législation. C'est à travers l'analyse de ces discours, des initiatives dirigées vers les acteurs locaux et de celles qui visent à faire évoluer la loi que nous rendrons saillants l'engagement patriotique et patrimonial du $\mathrm{TCF}$, son positionnement économique et enfin son investissement pour la préservation des montagnes et la sécurité de leurs habitants.

\section{De la protection du patrimoine à l'engagement sur la question forestière}

\section{Naissance du patrimoine naturel}

L'histoire du tourisme s'inscrit dans la longue durée (Boyer, 2000) et les attirances des touristes évoluent au fil des époques et des sensibilités (Corbin, 2001). Pourtant, le tournant du $X X^{\mathrm{e}}$ siècle est une période charnière pendant laquelle les événements s'accélèrent. L'avènement des loisirs (Corbin, 1995), sportifs notamment (Terret, 2010), est un catalyseur des pratiques touristiques. Le TCF est au centre de cette dynamique car il articule efficacement les initiatives sportives et touristiques (Schut et Levet-Labry, 2014), imposant une nouvelle façon de faire du tourisme (Bertho-Lavenir, 1999).

Les mobilités que l'on pourrait qualifier de «sportives ", à l'image du cyclotourisme ou du ski, amènent les touristes à traverser des espaces naturels pittoresques (Corbin, 2001) et à en apprécier les paysages. L'attrait pour la nature se renforce. À partir de là, les ressources touristiques ne sont plus simplement liées au patrimoine bâti mais également à la nature. Or, certains sites naturels sont de plus en plus convoités par des acteurs économiques pour des opérations diverses. Par exemple, l'engouement pour la houille blanche amène à détourner les cours d'eau qui produisaient les cascades les plus pittoresques pour les coiffer d'une conduite forcée métallique dévalant la montagne jusqu'à une turbine.

Aussi, le TCF prend à bras-le-corps la question de la préservation du patrimoine touristique : "Parmi les généreuses missions, dont le Touring Club s'est généreusement emparé nulle ne vaut, à notre idée, la protection des sites et des monuments » (Reclus, 1905a, p. 248). Une de ses productions les plus importantes consiste en un inventaire départemental des sites et monuments. Il a donné lieu à la publication d'une collection d'ouvrages parus entre 1902 et 1906 . Ce travail n'est pas vain car l'action du TCF est également tournée vers l'État pour produire le dispositif législatif susceptible de garantir durablement la protection des sites. Ainsi, le club contribue à la promulgation de la loi du 24 avril 1906 sur la protection des sites et monuments naturels de caractère artistique (Gauchon, 2002).

Dès lors, en identifiant des ressources naturelles pour le tourisme, l'État, par cette loi, s'investit dans le gouvernement de ces ressources. Par voie législative, il confère à une sélection de sites le caractère de bien public national. Leur détermination est confiée à des commissions 
départementales, qui vont pouvoir s'appuyer sur le travail d'inventaire réalisé par le TCF. La loi de 1906 révélera deux faiblesses qui restreindront ses effets : d'une part, les fortes disparités géographiques liées aux dynamiques plus ou moins volontaristes des acteurs locaux et, d'autre part, le faible pouvoir de coercition que la loi confère auxdits acteurs. Il faudra attendre la loi du 16 décembre 1930 pour que l'État s'octroie le droit de se passer de l'autorisation du propriétaire notamment.

\section{Préserver les bois et forêts}

La loi de 1906 permet de classer 459 sites et monuments naturels. Gauchon (2002) en établit une nomenclature : lacs, sources, cascades, gorges, cirques, rochers ou groupes de rochers, grottes, arbres ou groupes d'arbres et bois. S'il observe la prédominance des éléments aquatiques, c'est sur les deux dernières catégories que se focalise notre attention. L'arbre et les bois font également partie des éléments patrimoniaux comme l'atteste le discours d'Abel Ballif, président du TCF, qui voit dans les forêts « l'un des principaux attraits des voyages, l'une des gloires de la France » (Ballif, 1905, p. 532).

Leur fragilité face aux pratiques d'exploitation du bois incite le TCF à s'engager davantage pour leur préservation. Ainsi, à l'intérieur de son Comité des sites et monuments, est créée en janvier 1906 une commission des pelouses et forêts qui se concentre sur la problématique du reboisement : " la protection des sylves doit devenir l'une des grandes préoccupations du TouringClub dans sa lutte pour la beauté de la France » (Reclus, 1905a, p. 250). L'intérêt de cette commission est de pouvoir s'adjoindre le concours de personnalités compétentes et, pour certaines, déjà investies sur cette question. Sa composition est remarquable. En premier lieu, le sénateur Auguste Calvet. Avant d'être élu, ce notable, propriétaire, est convaincu qu'il est de son devoir moral de seconder « les efforts de ses paysans » (Kalaora et Savoye, 1986, p. 79). Il est à l'origine d'un syndicat de viticulteurs et réalise plusieurs missions officielles. À son entrée au Sénat, il devient spécialiste des questions agricoles et forestières. Il intègre ainsi en mars 1905 la commission chargée de réviser le code forestier, notamment pour répondre aux problématiques du déboisement ${ }^{2}$. Influencé par les théories leplaysiennes, il défend le reboisement pour permettre le développement économique et social local (Puyo, 1999). En parallèle, il anime diverses associations dont la Société forestière française des amis des arbres dont il occupe la présidence. Son vice-président, Jean-Honoré Audiffred, est également sénateur. Leur collaboration se prolonge naturellement jusque dans la commission du TCF. Là, un autre

2 Décret du 31 mars 1905 (reproduit dans Revue des Eaux et Forêts, 1905, 249). responsable associatif, Paul Descombes, président de l'Association pour l'aménagement des montagnes, se montre particulièrement dynamique pour défendre la cause du reboisement. À leurs côtés, on compte également des hauts fonctionnaires comme Léon Dabat, directeur de l'hydraulique agricole, deux anciens conservateurs et un inspecteur des Eaux et Forêts, Émile Cardot.

En soutenant une cause «patrimoniale " avec l'appui de ces personnalités, le TCF s'inscrit comme un nouvel acteur dans la controverse apparue à la fin du XVIII ${ }^{\mathrm{e}}$ siècle relative à l'effet de la forêt sur le régime des cours d'eau. Cette controverse au long cours oppose deux camps : les «ingénieurs » (essentiellement des ponts et chaussées) et les forestiers, chargés de la gestion et de la protection des forêts françaises (Corvol, 1987b). Ces derniers, fortement marqués par des conceptions romantiques, perçoivent la forêt comme jouant un rôle primordial, direct, non équivoque et bénéfique pour la protection contre les crues et inondations, la préservation des sols, la régulation du climat et la préservation des sources (Andréassian, 2004). Les positions des ingénieurs s'appuient sur des études plus scientifiques et méthodiques. Sans nier d'éventuels effets bénéfiques du reboisement sur le régime des cours d'eau, elles sont à la fois plus nuancées, plus hétérogènes et moins consensuelles que celles des forestiers, en mettant davantage l'accent sur le caractère plurifactoriel des phénomènes liés au climat et au régime des cours d'eau (Andréassian, 2004). Le TCF revendique explicitement, et à de nombreuses reprises, un parti pris forestier.

\section{Une action d'éducation d'envergure}

Rapidement, la commission des pelouses et forêts du TCF essaie de transmettre ses positions par une action d'éducation. Ce choix n'est pas surprenant. Parmi ses membres, le sénateur J.-H. Audiffred est connu pour son soutien aux mutualités scolaires forestières (Coincy, 1905) pour lesquelles E. Cardot avait lui-même rédigé un manuel d'appui technique incitant et facilitant leur création.

Cette action auprès du public scolaire concentre près de $90 \%$ des moyens, financiers notamment, du TCF ${ }^{3}$. Au cœur de ses investissements se situe la publication d'un livre, Le Manuel de l'arbre, qui est destiné à être distribué à tous les écoliers. Écrit par E. Cardot, édité par le TCF, il

\footnotetext{
3 Lors d'un bilan financier sur les trois premiers exercices de la commission, le TCF fait état de 86775 francs de dépenses qui se ventilent en 26070 francs de subventions, dont les deux tiers sont destinés à des sociétés forestières scolaires, 5705 francs de récompenses distribuées lors des Fêtes de l'arbre et 55000 francs pour l'édition du Manuel de l'arbre, du Manuel de l'eau et de supports pédagogiques à destination des écoles (source: Defert, 1909a, p. 64-65).
} 

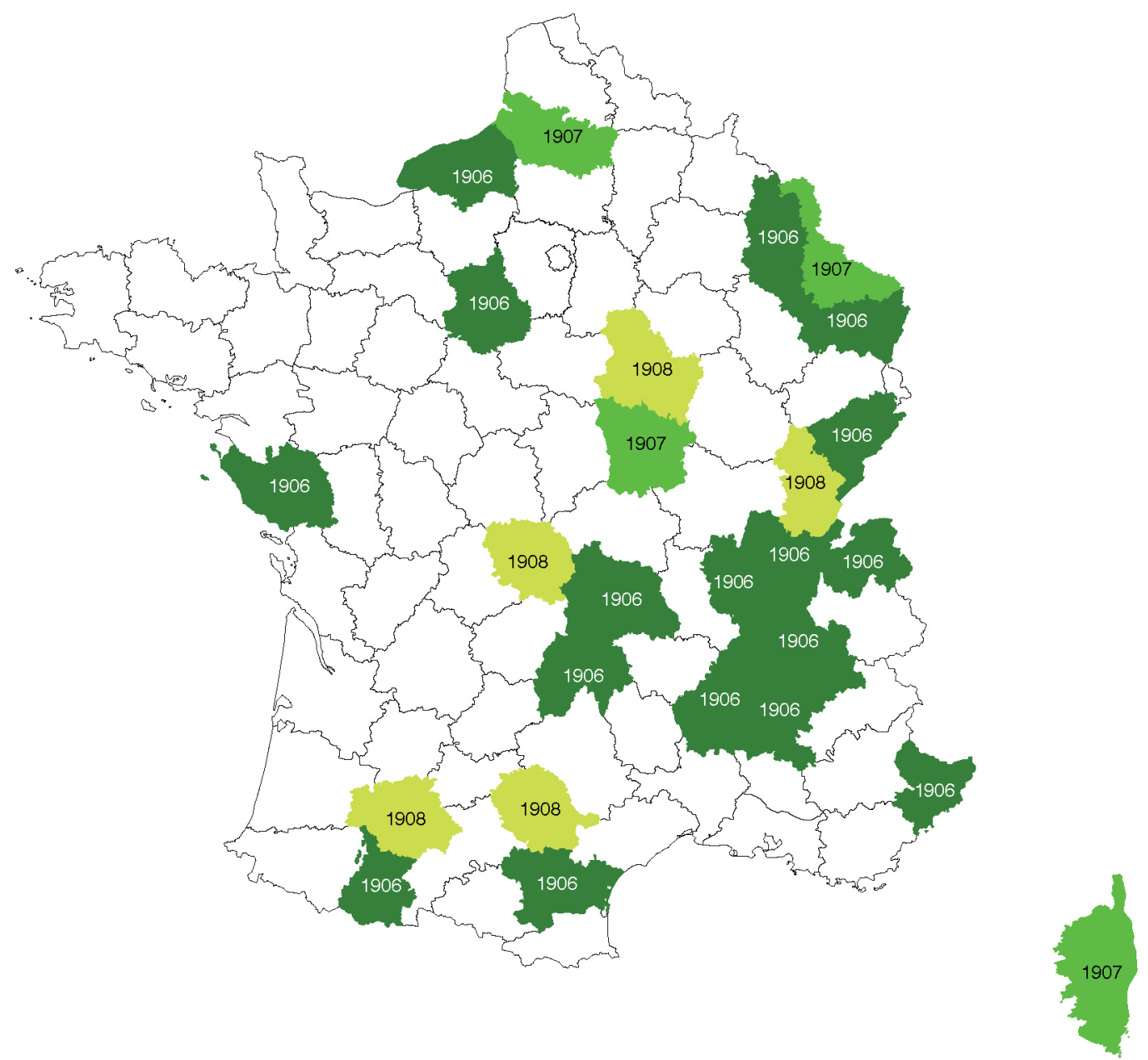

Carte. Fêtes de l'arbre organisées entre 1906 et 1908.

paraît en 1907 avec un premier tirage à 50000 exemplaires. L'une des missions de ce Manuel est de transmettre à la jeunesse « l'amour de l'arbre » (Cardot, 1907a, p. 5). Pour accompagner la propagande visée par cet ouvrage, le TCF participe à la multiplication de Fêtes de l'arbre. Ces événements, déjà mis en place en Italie ou aux États-Unis sous l'appellation d' « Arbor Day », sont des occasions de partager une admiration pour les beautés forestières et de favoriser les initiatives locales. Un nombre croissant de départements se trouve impliqué chaque année, notamment ceux des différents massifs français (Carte).

Compte tenu de leur relative fragilité, soit parce que le bâti est ancien et dans certains cas déjà à l'état de ruine, soit parce que l'environnement naturel est menacé par des projets d'aménagement liéà l'urbanisation croissante ou à l'exploitation industrielle des ressources du territoire, le TCF s'engage dans une politique de protection de ces sites et monuments. Dans ce dispositif, l'arbre, les bois et les forêts prennent une place à part entière car ils représentent un attrait touristique important aux yeux de ses dirigeants.
Or, le problème du déboisement est ancien, au point même de faire apparaître le TCF comme un tard venu dans sa prise en charge. La déforestation est déjà condamnée au début du XIX ${ }^{\mathrm{e}}$ siècle sous des plumes célèbres, comme celle de Chateaubriand : « Partout où les arbres ont disparu, l'homme a payé son imprévoyance » (cité par Cardot, 1907a, p. 85). Entre 1860 et 1890, la question du reboisement prend une place importante sur la scène publique. En 1860, une loi est promulguée mais ne s'avère pas suffisamment efficace pour clore le débat. La question revient à l'agenda politique et une nouvelle loi renforce le précédent texte en 1882. Le Gouvernement prend à ce moment en charge la ressource forestière avec l'aide des communes. Après quelques années de tensions, la question du reboisement s'apaise et quitte peu à peu le devant de la scène politique, mais le TCF critique, même durement, les choix de répartition des dépenses publiques : "Que de ruines évitées, que de richesses créées par un emploi plus judicieux de l'argent des contribuables! Et pas une voix ne s'est élevée dans la Chambre ou au Sénat pour protester contre de tels errements ! »(Defert, 1907a, p. 197). Ainsi, le TCF s'investit 
dans une mission qui relève des acteurs publics mais qui pour lui est une cause légitime du point de vue du tourisme et donc de ses activités, avec l'ambition de pallier l'insuffisance de l'État.

On le voit, la déforestation est une question complexe au centre de multiples enjeux. Le tourisme n'est probablement pas le plus important socialement. Or, le TCF est habitué à défendre les intérêts économiques des initiatives qu'il loue ou qu'il impulse directement. Aussi, le débat s'engage sur cette voie pour convaincre et également participer à une nouvelle gestion des espaces forestiers.

\section{Investir dans la forêt}

\section{L’enjeu économique du déboisement/reboisement}

Dans son argumentaire, le TCF énonce ouvertement que la forêt est une ressource naturelle importante du point de vue économique et qu'il convient de l'entretenir et de la développer pour ne pas appauvrir le pays. Comme le répète le Manuel de l'arbre: «Les peuples qui aiment les forêts sont laborieux et prévoyants. En raison de leurs mœurs et de leurs traditions, ils conservent et accroissent le capital, quelle que soit sa forme, qui constitue le matériel de leur civilisation. Ce sont les peuples en progrès. » (Ernest Guinier cité dans Cardot, 1907a, p. 86). Or, le bois des forêts sert de nombreuses industries. Le Manuel de l'arbre le rappelle opportunément, pour montrer que la connaissance est acquise de longue date mais que le débat n'est pas clos : «De la conservation des forêts dépendent le succès de l'agriculture, du commerce, des manufactures et des arts, la marine, la navigation intérieure, les mines, toutes les commodités de la vie et notre existence même » (Rapport fait en l'an IV à la Convention, cité dans Cardot, 1907a, p. 85). Le bois est donc nécessaire pour le fonctionnement économique de la société dans son ensemble.

La forêt est aussi un capital, qu'il est possible de développer et de transmettre à ses enfants car il prend de la valeur à long terme. «Certains cantons de ces sapinières ont une valeur de 15000 et 30000 à l'hectare qui ne valaient pas 500 francs il y a cent ans » peut-on lire dans le Manuel (Cardot, 1907a, p. 66). Aussi, il est important pour le pays de préserver ses richesses en ne réduisant pas sa surface forestière.

En réalité, la forêt représente un bien économique à plusieurs titres : en tant que stock de bois qui constitue un véritable capital pour la population, mais également par son rôle régulateur dans la circulation des eaux. De ce fait, elle a un impact positif sur l'agriculture qui bénéficie d'une irrigation plus régulière. Aussi, l'entretien et donc le développement du capital forestier sont importants pour l'enrichissement du pays. Ce discours n'accable pas l'exploitation sylvestre mais condamne ses excès.

En effet, le TCF explique qu'il y a deux façons d'exploiter un bois : l'une, raisonnable, qui anticipe sur les besoins futurs et qui consiste à pratiquer des coupes partielles pour permettre à la forêt de se régénérer ${ }^{4}$, et l'autre, critique, qui, sacrifie un espace boisé pour un enrichissement ponctuel et qui consiste à réaliser des coupes rases. Le TCF, sous la plume de Henry Defert, $\mathrm{s}$ 'indigne de ces procédés et n'hésite pas à condamner ouvertement ses responsables. Par exemple, des sociétés d'exploitations forestières sont montrées du doigt :

"Société d'exploitation forestière au capital de 1600000 francs, ayant pour objet la réalisation et l'exploitation de forêts en France et à l'étranger, notamment celle de la forêt de Marchenoir ! Et, pour que nul ne s'y trompe, la publication annonce que la société se constitue avec un matériel de destruction formidable : scies verticales, scies à ruban, scies à lames multiples, scies circulaires, trucks, machines à vapeur et électriques, voie ferrée Decauville, forge, cité ouvrière, en un mot, toute la lyre des engins de dévastation et de ruine! » (Defert, 1908, p. 8).

Ce défrichement est souvent l'occasion d'une réaffectation du sol. En effet, les forêts n'offrent pas les mêmes atouts que les prairies pour servir de pâturages ; c'est pourquoi les coupes rases seraient pratiquées pour augmenter les surfaces dédiées à l'élevage, croient devoir dénoncer le TCF.

«On pourrait se consoler de la disparition des grands bois, en pensant que si, en somme, nous n'avons plus de forêts, nous avons une compensation dans l'abondance des produits pastoraux. Mais il n'en est rien. Bêtement le pasteur a tué la poule aux œufs d'or. Tandis que le rendement des pâturages de l'Est s'élève jusqu'à 100 francs, pour la période d'été, les profits correspondants ne sont dans les Pyrénées que de 10 et même 5 francs. Le seul département du Doubs livre annuellement au commerce des fromages pour une valeur de sept millions, tandis que les départements pyrénéens, d'un bout à l'autre de la chaîne, ne fournissent pas, en qualité médiocre, de quoi subvenir à la consommation locale. » (Defert, 1907b, p. 55).

La critique du pastoralisme est récurrente dans les colonnes de la Revue mensuelle du TCF, et même souvent virulente. Elle ne heurte pas ses membres qui sont majoritairement issus des couches aisées et citadines de la population, réceptives au discours sur l'imprévoyance et l'archaïsme de la paysannerie, les agriculteurs, lorsqu'ils sont additionnés aux ouvriers, ne représentant que 0,7 à $1 \%$ des demandes d'adhésion au TCF avant la Première Guerre mondiale (Larique, 2006).

\footnotetext{
4 Une forme de quotas est envisagée par Henry Defert: laisser debout un minimum de 100 arbres par hectare d'un diamètre de $20 \mathrm{~cm}$ (Defert, 1909a, p. 63).
} 


\section{Encouragements pour l'exploitation forestière}

Condamner les mauvaises pratiques n'est ni suffisant, ni réellement efficace. Le club compte parmi ses adhérents des entrepreneurs, des commerçants, des bourgeois aisés capables d'investir dans une exploitation rentable. Aussi, les membres du TCF actifs dans la cause du reboisement tentent d'orienter les investissements des adhérents, de mobiliser des capitaux pour s'engager dans une exploitation raisonnée de la forêt. Pour cela, le meilleur moyen de les convaincre consiste à leur faire la démonstration, chiffres à l'appui, du rendement des forêts. En Franche-Comté, les sapinières qui sont très rentables sont prises en exemples par H. Defert :

« D'autres forêts de la même région ont donné pendant une période de 35 ans environ, de 1860 à 1895, un rendement annuel moyen à l'hectare sensiblement supérieur puisqu'il ne s'est pas élevé à moins de 116 francs. Quelquesunes mêmes, particulièrement bien aménagées, ont produit de 127 à 170 francs par hectare et par an ; et cela, sans dépasser une seule fois la possibilité de la forêt, c'est-à-dire la production ligneuse annuelle, de telle sorte que ces magnifiques rendements peuvent se maintenir indéfiniment » (Defert, 1907c, p. 542).

Ces revenus d'exploitation s'ajoutent à la constitution d'un capital puisque les forêts entretenues représentent une valeur économique importante. Là encore, les chiffres mis en valeur parlent d'eux-mêmes, H. Defert évoque le cas de la forêt de Bon dans le Doubs qui aurait été mise en vente par l'État à 30000 francs. N'ayant pas trouvé acquéreur, la forêt est restée publique. En trente ans, elle a rapporté 1079380 francs et représente, selon l'auteur, une valeur totale en matériel ligneux de l'ordre du million de francs (Defert, 1907c, p. 542). Ces exemples étayent une démonstration qui doit convaincre les individus d'orienter leurs investissements vers les exploitations forestières.

En plus de l'initiative privée, le TCF cherche auprès de l'État un soutien financier. À cette époque, le système des retraites est capitalistique. Aussi, les caisses de retraites disposent d'importants capitaux qu'elles ont intérêt à faire fructifier à long terme. E. Cardot étudie l'hypothèse et montre l'intérêt économique pour les caisses de retraites ouvrières d'investir dans la cause du reboisement :

«Au point de vue général, la création de 10000 hectares de forêts productives aboutirait au bout d'un siècle à la formation d'un capital forestier qui, nonobstant les réalisations, pourrait avoir une valeur capitale de 2 à 3 milliards. Ainsi on aurait renforcé le crédit de l'État tout en contribuant à assurer le succès d'une œuvre sociale telle que l'œuvre des Retraites ouvrières et à développer l'un des éléments les plus essentiels de la prospérité publique. » (Cardot, 1907b, p. 130).
Le sénateur J.-H. Audiffred, également membre de la commission du TCF, porte cette suggestion dans les plus hautes instances. En 1910, au Sénat, la proposition soulève des réticences, mais J.-H. Audiffred défend à plusieurs reprises ce texte qui prévoit que les caisses de retraites ouvrières puissent réaliser des placements en forêts ou terrains à reboiser jusqu'à concurrence d'un $400^{\mathrm{e}}$. Le sénateur connaît bien ces problématiques car il a participé, en tant que député, aux commissions sur la caisse nationale des retraites dès les années 1880. Le vote entérine finalement cette proposition.

\section{Le soutien des politiques publiques?}

Parmi les arguments avancés au Sénat, deux avaient touché l'auditoire : 1) le risque du placement lié aux incendies ; 2) la faible rentabilité du placement. Le sénateur J.-H. Audiffred a su répondre au premier argument en rappelant que seuls les résineux sont affectés par les incendies et qu'ils sont assurés contre ce risque, mais le deuxième point est plus délicat. En effet, les élus savent que le poids de la fiscalité est particulièrement désavantageux pour l'exploitation forestière ${ }^{5}$.

Au sein du TCF, cette problématique est également bien identifiée. Une analyse détaillée du régime fiscal des forêts permet de mettre en évidence que les propriétaires des bois paient de lourdes charges dans la perspective d'un revenu incertain à long terme. Autrement dit, le choix le plus rentable serait la coupe rase immédiate de toutes les parcelles forestières. H. Defert donne l'exemple d'un sociétaire, propriétaire de trois hectares de taillis. Le système fiscal lui fait payer 153,68 francs d'impôt par an, soit 7684 francs pour 50 ans. Or, l'estimation des revenus dégagés par cette parcelle est estimée par l'auteur à 1900 francs (Defert, 1910, p. 246). Aussi, le meilleur conseil fiscal serait de procéder à une coupe rase pour mettre en location son terrain pour la culture, effet justement combattu par le TCF. Dès lors, le club exprime l'intérêt d'une réforme fiscale moins dissuasive, sinon incitative :

"Que d'initiatives fécondes, que de bonnes volontés agissantes dont profiterait le reboisement, si, au lieu de considérer la question de l'impôt forestier au point de vue étroit de la fiscalité, le législateur se décidait à la régler dans le sens de l'intérêt général du pays, de sa prospérité économique et de sa beauté !» (Defert, 1911, p. 200).

E. Cardot (1911) rédige des propositions d'évolution du système fiscal afin d'adapter le dispositif aux particularités de l'exploitation forestière. Le point central est lié au calcul de l'impôt qui devrait s'effectuer uniquement sur la valeur du produit ligneux issu des coupes. De cette manière, le propriétaire n'aura pas à avancer l'impôt sur

\footnotetext{
5 Intervention du sénateur Fortier au Sénat à la séance du 25 janvier 1910 (Sénat, 1910, p. 104).
} 
un revenu futur de son capital forestier. Le second point relève toujours de la question fiscale davantage pensée comme une mesure incitative. E. Cardot propose une défiscalisation des jeunes bois créés de main d'homme pour une durée de trente ans. Dans la période étudiée, ces propositions n'aboutiront pas à des mesures concrètes entérinées par le ministre des Finances.

En dépit des difficultés du TCF à voir aboutir l'ensemble de ses demandes, il est clairement perceptible, à travers la plume de H. Defert, qu'il y a une volonté de marier l'intérêt pour le reboisement avec les intérêts économiques de l'ensemble des acteurs publics et privés. Outre ses efforts pour mobiliser les investissements des particuliers ou des caisses de retraites notamment, le TCF incite également les pouvoirs publics à investir dans le reboisement.

En effet, l'État peut y trouver une autre forme de rentabilité : la forêt peut réduire les catastrophes naturelles telles que les inondations et glissements de terrain et ainsi lui éviter de dépenser chaque année des millions de francs pour aider les victimes. De ce point de vue, $H$. Defert s'indigne du fonctionnement de l'administration publique :

«La conspiration du silence s'est faite comme par enchantement pour creuser sans phrase ce nouveau trou dans le budget, qui n'a même pas l'excuse d'un dédommagement sérieux pour les victimes de calamités qu'il serait si simple d'empêcher à beaucoup moins de frais ! Là-dessus, il ne peut y avoir qu'un cri : C'est bête à pleurer ! » (Defert, 1907a, p. 197).

La question du reboisement est donc une question économique importante mais pas seulement car elle recouvre d'autres enjeux, notamment pour les pouvoirs publics.

\section{Porter la cause du reboisement et protéger la montagne}

\section{La forêt : une protection contre les crues et les inondations}

Pour porter la cause de la protection des bois et forêts, le TCF mobilise un argumentaire prudentiel et interpelle l'État dans l'exercice de ses missions : la prise en charge des effets dévastateurs des crues et inondations. Cet enjeu vient appuyer avec force ses revendications.

En associant directement le reboisement au régime hydrologique, le TCF reprend l'argumentaire des forestiers. Il mobilise également les travaux de certains ingénieurs, notamment Alexandre Surell (1870), qui a démontré le rôle bénéfique des forêts pour la conservation des sols. Mais, là où A. Surell était nuancé sur les autres bienfaits des arbres, le TCF affirme leur importance pour limiter les phénomènes de la formation de torrents et les crues en aval, pour réguler le régime des pluies ou encore la température de l'air. C'est toutefois l'argument de la protection contre les crues qui est le plus récurrent, ce qui conduit d'ailleurs le TCF à disqualifier le discours de ceux qui remettent en question cette influence bénéfique des forêts. L'argumentaire s'inscrit dans un discours souvent alarmiste, sur le mode de la prophétie de malheur : "Il y a urgence, urgence extrême. Il faut à tout prix enrayer, sinon gare à la traînée de poudre » (Detois, 1906, p. 357). La situation des montagnes serait déjà catastrophique, et deviendrait sous peu irréversible si aucune mesure n'était prise pour lutter contre le déboisement.

Les exploitants et marchands de bois, fustigés pour leur gestion économique à court terme du patrimoine forestier, les communes, critiquées parce qu'elles sont incapables de protéger leurs forêts, voire parce qu'elles se rendent complices de leur déclin, et l'État, à cause de son système $\mathrm{d}$ 'imposition qui incite au déboisement, sont la cible de nombreuses critiques : la mauvaise gestion économique des bois et forêts, selon le TCF, avec pour corollaire une réduction des surfaces boisées, entraînerait un risque de crues torrentielles.

C'est toutefois vers un autre responsable que pointe, de façon récurrente et virulente, la plupart des charges critiques du TCF : le pastoralisme. La déforestation due aux moutons (notamment transhumants), par la faute des «montagnards ", est dénoncée. Les bergers pyrénéens font figure de mauvais élèves. On peut ainsi lire dans les colonnes de la revue :

« C'est surtout le pastorat qui les ruine [les Pyrénées], le pastorat insouciant, ignorant, imbécile, fatal tel que le comprirent les patriarches de l'Orient continués jusqu'à nos jours par les nomades aussi peu capables de comprendre l'enchaînement des faits qu'ils le sont de se fixer définitivement quelque part et de muer enfin leur tente en maison. Les «moutonniers » pyrénéens méritent deux fois ce nom; comme bergers de moutons et de brebis et comme hommes emprisonnés dans les plus noirs cachots de la tradition » (Reclus, 1905b, p. 436).

Cette critique des montagnards, dont les moutons dévorent gazons et forêts, constitue un discours qui s'intensifie et tend à se généraliser durant toute la deuxième moitié $\mathrm{du} \mathrm{XIX}^{\mathrm{e}}$ siècle parmi les naturalistes, les géographes, ou encore les forestiers pyrénéens condamnant « l'incurie pastorale » (Briffaud et Métailié, 1987). Ce discours va « culminer dans les années 1900-1910, où il est exprimé dans les colloques, les revues locales ou nationales, les journaux » (Métailié, 2006, p. 196).

La dégradation pastorale, assénée plus que démontrée (Métailié, 2006), fait ici figure de bouc émissaire. Ce positionnement intellectuel est difficilement dissociable 
d'un positionnement social. Pour Peltier (2005), outre les intérêts catégoriels des forestiers qui cherchent à préserver leur rôle et leur influence, le discours vise à assurer la sécurité de la plaine face aux crues et aux populations des montagnes. Pour l'auteur, « La volonté de protection de la plaine est révélatrice du fait que la théorie du déboisement est la résultante d'une vision avant tout citadine de ce qu'est la montagne - et surtout de ce qu'elle devrait être » (Peltier, 2005, p. 130). Il n'est pas étonnant de retrouver ce discours au sein du TCF, dont les membres sont à cette époque essentiellement des citadins aisés (Larique, 2006 ; Cousin et Réau, 2009), amateurs de tourisme.

\section{La stratégie de diffusion du Manuel de l'arbre}

L'action du TCF en faveur de la restauration des surfaces boisées se fait à plusieurs niveaux : c'est à la fois en s'assurant une audience auprès des plus hauts responsables de l'État et au plus près des populations des montagnes que le club veut faire porter sa cause.

Il faut préciser que la cause du reboisement, même si elle n'a plus l'écho qu'elle a connu jusqu'aux années 1880, est loin d'être confinée au TCF ; en effet, « à la suite de $1^{\prime}$ Exposition universelle de 1889, on assiste à l'émergence de plusieurs associations privées se consacrant à la promotion du reboisement du sol de la patrie » (Puyo, 1999).

Dans l'enseignement également, cette problématique de la gestion des forêts françaises a également fait l'objet d'une certaine attention. Terral (2008) rapporte les interventions de Marcel Charlot (inspecteur général de l'enseignement primaire) qui plaide dans la Revue pédagogique en 1901 pour une meilleure considération de l'arbre, et de Paul Lapie (universitaire devenu recteur de l'Académie de Toulouse en 1911) qui plaide un peu plus tard pour un enseignement sylvopastoral à l'école primaire. La préoccupation à l'égard de la déforestation et du reboisement ne s'est jamais complètement effacée chez les acteurs publics.

$C^{\prime}$ est dans ce contexte favorable à la diffusion des idées du TCF que paraît le Manuel de l'arbre, contexte d'autant plus favorable que l'association, reconnue d'utilité publique en 1907, " apparaît comme une institution très recommandable » (Terral, 2008). Dans le souci "patriotique d'instruire la jeunesse et de préparer la mentalité des générations futures ${ }^{6} »$, E. Cardot y présente les bienfaits de l'entretien des forêts et des pâturages tant pour l'environnement que pour l'homme. La lutte contre les crues et inondations et les effets dévastateurs du pastorat irréfléchi sont très présents dans l'ouvrage. L'impact des troupeaux qui dévorent les

\footnotetext{
6 Avant-propos d'A. Ballif dans le Manuel de l'arbre (Cardot, 1907a, p. 3).
}

forêts, favorisant les phénomènes de ravinement et la formation de torrents y est plusieurs fois illustré à l'aide de photographies. La diffusion du manuel s'appuie sur deux stratégies complémentaires : d'une part, investir le maillage social au plus près des citoyens et des organes de décision locaux, et d'autre part, s'assurer le soutien des organes de décision plus « centraux ». Afin de favoriser une diffusion large et rapide, l'ouvrage est proposé gratuitement aux maires et instituteurs qui en font la demande.

Cette entreprise bénéficie du soutien du ministère et de l'inspection de l'Enseignement primaire. Le manuel reçoit en effet un accueil très positif de la part du ministre. Son avis, favorable, est publié en 1907 dans la revue du TCF et, en 1909, il remercie le club pour avoir mis à disposition 50000 exemplaires de cet ouvrage. Avec son approbation, le TCF se lance dans une large campagne d'information sur la mise en place de fêtes de l'arbre en envoyant un courrier à tous les inspecteurs d'Académie.

Cette volonté du TCF trouve un écho du côté de l'instruction publique. Ainsi, la revue publie en 1909 la lettre de M. Hébré, inspecteur du primaire, adressée aux instituteurs, dans laquelle il demande non seulement d'organiser un enseignement sylvopastoral, citant au passage l'œuvre du TCF, mais aussi de mettre sur pied les "sociétés forestières scolaires " souhaitées par le club à l'occasion des fêtes de l'arbre (Defert, 1909b, p. 112). À leur niveau, les maîtres et les écoliers sont ainsi encouragés à porter la cause du reboisement, notamment en gérant eux-mêmes une pépinière afin de boiser ou reboiser leur commune.

\section{Une présence locale via des incitations financières}

Le TCF n'hésite pas à intervenir localement, au risque de disperser ses moyens somme toute limités. En réalité, ce mode d'action fonctionne parce qu'il s'agit essentiellement de communication, c'est-à-dire d'une part, de la transmission d'un message, mais également $d^{\prime}$ 'un plaidoyer des bonnes pratiques, de la condamnation des actions jugées néfastes et du soutien, souvent financier, par voie de subventions, aux initiatives locales positives. Ce dernier aspect, même s'il a une dimension tangible par l'allocation de ressources financières ou matérielles, est surtout un dispositif de communication puissant. En effet, les subventions du TCF sont la plupart du temps relativement modestes (souvent quelques dizaines ou centaines de francs) et le budget de la Commission des pelouses et forêts est totalement dérisoire par rapport à l'argent public engagé dans cette cause. Mais ces soutiens permettent de faire émerger de bonnes pratiques, d'assurer les acteurs du soutien du club et de valoriser leurs initiatives. Ces valorisations ciblent l'action de particuliers, des mairies ou des associations forestières scolaires. 
Parmi elles, il faut citer l'Association pour l'aménagement des montagnes, fondée par P. Descombes. En louant pour cinq ans le pâturage de deux communes des Pyrénées, cette association a restreint la présence des troupeaux pour endiguer la dégradation des terrains fréquentés jusqu'alors par les 800 bêtes des deux villages et les 3000 moutons espagnols en transhumance. Elle met en œuvre des actions de plantation, d'embroussaillement des roches et d'épandage d'engrais. Sa " noble entreprise » (Reclus, 1905b, p. 437) est soulignée dans les colonnes de la revue, et ce «bon exemple » (Ballif, 1905, p. 534) est cité jusqu'à l'assemblée générale de 1905 en présence de M. Dariac, directeur de cabinet représentant le ministre de l'Agriculture. De la même façon, le conseil d'administration du TCF (1906, p. 85) vote des félicitations à des particuliers comme M. Longchampt pour les opérations de reboisement qu'il a exécutées sur plus de 130 hectares, aux environs de la commune de Hautepierre dans le Doubs. Les associations scolaires et les maîtres qui ont contribué, d'une façon ou d'une autre, au reboisement à leur échelle sont régulièrement cités en exemple et reçoivent des prix et des subventions.

\section{Une action sur le cadre législatif}

L'action du TCF est ambitieuse et ne saurait se limiter aux possibilités financières de l'association. Il doit ses réussites à l'effet de levier qu'il sait donner aux initiatives qu'il soutient. Aussi, non content de convaincre les entrepreneurs de s'engager dans la voie de l'exploitation forestière, le TCF cherche à impliquer l'Etat et ses moyens. Il s'agit bien ici d'une mobilisation et non d'un assentiment comme le club a su l'obtenir très tôt du ministre de l'instruction publique pour la diffusion du Manuel de l'arbre. En effet, le TCF souhaite impliquer les services des agents des Eaux et Forêts pour gérer les domaines forestiers, y compris ceux qui ne sont pas publics. Il souhaite également que les associations - comme le TCF ou ses partenaires, par exemple l'Association pour l'aménagement des montagnes et la Société forestière française des amis des arbres - puissent acquérir des biens forestiers pour ainsi leur permettre de participer activement au reboisement ou à la préservation d'espaces forestiers. De plus, compte tenu de la première disposition souhaitée, ces associations pourraient s'appuyer sur les agents d'État pour assurer la gestion de ces bois. Pour obtenir de l'État un tel engagement, le TCF doit œuvrer auprès des élus du gouvernement et convaincre.

Pour persuader les députés et sénateurs, la prise en compte du risque d'inondation est encore et toujours au cœur de l'argumentaire. En 1907, le TCF publie dans sa revue une proposition de loi intitulée : « Forêts de protection » (Commission des pelouses et forêts du TCF, 1907). Après la catastrophe de Bozel, le 16 juillet 1904, et celle des Fourneaux, le 23 juillet 1906, au cours desquelles ces villages savoyards ont été ravagés par de violentes coulées de boue et de gravats, ce discours ne peut laisser les élus insensibles puisqu'ils ont été amenés à voter des crédits pour l'aide aux victimes ${ }^{7}$.

Le député de Haute-Savoie, Fernand David, qui a probablement le souvenir de la catastrophe qu'a connue, sur son territoire, la commune de Saint-Gervais-les-Bains en 1892, s'engage avec le TCF. Très sensible aux questions liées à l'agriculture, il participe activement à la Commission de l'agriculture et critique à maintes reprises l'action du ministère de l'Agriculture qui, selon lui, manque d'efficacité. Son engagement en faveur du reboisement est associé à des propositions sur la restauration des terrains de montagne ou encore sur l'enseignement agricole. Il trouve dans le TCF un partenaire ${ }^{8}$. Dès la fin de l'année 1906, alors qu'il rapporte le budget du ministère de l'Agriculture, il fait l'éloge à la Chambre de la « croisade » entreprise par le TCF (Defert, 1906, p. 544). L'année suivante, il rédige plusieurs projets de lois qui convergent avec ceux du club dont il se fait le bras armé puisque la dernière proposition, déposée le 2 décembre 1907, n'est autre que le texte proposé par le TCF.

Ce soutien à la chambre est doublé au Sénat. En effet, le TCF peut compter sur l'énergie déployée par le sénateur J.-H. Audiffred. Membre de la première Commission des pelouses et forêts, il est le personnage-clé de l'aboutissement de l'initiative législative du club. Il se saisit de toutes les occasions pour faire avancer le projet de loi. À la suite de la crue exceptionnelle de la Seine en 1910 et des inondations qu'elle a entraînées, il interpelle l'Assemblée sur la question du reboisement : " Je demande à interpeller le Gouvernement : $1^{\circ}$ sur les mesures qu'il compte prendre pour prévenir les désastres des inondations, par l'emploi combiné des moyens actuellement connus, reboisements, construction de barrages, réservoirs, établissement de digues insubmersibles... » (Sénat, 1910, p. 96). Il obtient pour les dirigeants du TCF une rencontre avec le ministre de l'Agriculture en 1911 afin d'avoir son soutien dans le vote du projet de $\operatorname{loi}^{9}$. Finalement, sa ténacité lui permet d'obtenir gain de cause : le 3 juin 1913, le texte est adopté.

\footnotetext{
7 Le 29 décembre 1904, une loi fut votée ouvrant un crédit supplémentaire de 50000 francs pour aide aux victimes de la catastrophe de Bozel en Savoie (Journal Officiel, 31 décembre 1904).

8 Entre 1911 et 1912, Fernand David, alors ministre du commerce et de l'industrie, travaille en étroite collaboration avec le TCF à la création de la première école hôtelière dans la ville de Thonon-les-Bains, en Haute-Savoie.

9 Malheureusement, une succession de remaniements ministériels (sept ministres se succèdent entre 1910 et 1913) réduit les effets de cette visite.
} 
Il a subi quelques transformations mais conserve ses fondements : il permet de mobiliser l'administration forestière pour gérer des domaines privés et ouvre aux associations et caisses d'épargne la possibilité d'investir dans le reboisement et l'exploitation forestière. Ainsi, le TCF obtient le droit de défendre directement sa cause en participant à l'action publique liée au reboisement et réussit à mobiliser l'appareil législatif grâce à un lobbying bien ciblé. De cette façon, il implique des partenaires privés et l'administration forestière dans la gestion et la préservation des forêts. En s'engageant dans cette cause nationale, il sert également ses propres intérêts puisqu'il peut, en tant qu'association reconnue d'utilité publique, utiliser ses fonds pour acquérir des biens forestiers. Ainsi, fort d'un legs conséquent évalué à 320000 francs ${ }^{10}$ qui s'ajoutent à ses ressources propres, le TCF se donne l'opportunité de s'affirmer comme un acteur de poids des questions forestières. Mobilisé par la création au même moment du premier parc national à La Bérarde, le club peut dès lors accompagner l'initiative qui consiste à créer une caisse pour financer l'entretien des parcs. Il s'agit de soustraire au pastoralisme les vallées du massif du Pelvoux et d'engager des actions de reboisement dans un lieu que le TCF a toujours valorisé (Schut, 2013), et dont il a facilité l'accès pour favoriser son développement touristique.

En portant la cause du reboisement, le club s'investit dans une mission à la charge des acteurs publics, agissant dans une diversité $\mathrm{d}$ 'arènes pour parvenir à ses fins : des municipalités aux écoles et jusqu'aux délibérations parlementaires, le TCF multiplie les sollicitations. Il n'en reste pas moins difficile, en dehors de son impact sur la législation, de mesurer l'efficacité d'un certain nombre d'actions de l'association. La véritable portée incitative des subventions et prix accordés, ou encore l'utilisation concrète qui a été faite du Manuel de l'arbre ne peuvent pas être facilement évaluées.

\section{Conclusion}

Le TCF s'est donné pour mission de développer le tourisme sous toutes ses formes. Son action protéiforme $\mathrm{s}^{\prime}$ inscrit dans une logique globale qui s'avère in fine efficace. L'exemple de l'investissement du club dans la lutte contre le déboisement en est une illustration. Le point de départ est clairement lié à la préservation des sites pittoresques de France. À partir de là, la défense des sites forestiers et montagnards passe par sa participation à une cause nationale : le reboisement. Dès lors, le TCF s'engage pleinement dans cette direction, usant de ses capacités de persuasion auprès des hautes sphères de

\footnotetext{
${ }^{10}$ Legs Janssen « destiné à faire des expériences de reboisement » (Conseil d'administration du TCF, 1913, p. 491).
}

l'État pour utiliser les ressources financières des caisses de retraites, ou mobilisant son réseau sur l'ensemble du territoire pour multiplier les actions locales, dans les écoles notamment.

La question du reboisement est déjà ancienne et, de cette façon, le TCF tente de relancer le débat dans l'espoir $\mathrm{d}^{\prime}$ obtenir davantage de résultats sur le terrain. Ses efforts se concrétisent dans de très nombreuses actions locales, dont il est toutefois difficile de mesurer la portée. Ils conduisent également à une évolution législative sensible qui offre des possibilités supplémentaires pour multiplier les initiatives en faveur du reboisement.

Fidèle à ses modes d'action, le TCF réussit, avec peu de moyens, à jouer de son influence auprès des élus qui soutiennent et portent son projet. Les membres de l'association cherchent également à sensibiliser et à mobiliser des acteurs privés. Chacune de ces actions est susceptible de contribuer à la préservation des ressources et par extension des paysages boisés.

Or, c'est bien le paysage pittoresque qu'il convient de sauver, et les efforts du TCF, à la même époque, en faveur de la protection du paysage, aboutissent également à la promulgation d'une loi. Le texte de 1906 s'avère une étape importante avant celui de 1930, qui confère à l'État des moyens plus efficaces dans la réalisation de ses missions. Le TCF attire l'attention sur la problématique du reboisement. Néanmoins, la Guerre met un sérieux frein à ces actions, à l'image du développement des parcs nationaux dont le premier est créé à la veille de la Guerre et sera sans suite après l'Armistice. La question des parcs nationaux ne reviendra qu'en 1960. Il en va de même pour celle du reboisement qui connaîtra alors un regain d'intérêt, dans un contexte différent.

\section{Références}

Andréassian V., 2004. Couvert forestier et comportement hydrologique des bassins versants, La houille blanche, 2, 31-35.

Ballif A., 1905. Discours prononcé lors de l'assemblée générale de 1905, Revue mensuelle du TCF, décembre, 530-535.

Bertho-Lavenir C., 1999. La roue et le stylo. Comment nous sommes devenus touristes, Paris, Odile Jacob.

Boyer M., 2000. Histoire de l'invention du tourisme dans le Sud-Est de la France XVI ${ }^{e}-X I X^{e}$, La Tour d'Aigues, L'Aube.

Briffaud S., Métailié J.-P., 1987. Du vert paradis aux affreux déserts. Perceptions du paysage pastoral pyrénéen aux $\mathrm{XVIII}^{\mathrm{e}}$ et $\mathrm{XIX}{ }^{\mathrm{e}}$ siècles, in Association française de géographie physique, Paysages, aménagement, cadre de vie. Mélanges offerts à Gabriel Rougerie, Paris, AFGP, 129-138.

Buttoud G., 1983. L'État forestier : politique et administration des forêts dans l'histoire française contemporaine. Thèse d'état en sciences politiques, Université de Nancy II, Nancy.

Cardot E., 1907a. Manuel de l'arbre, Paris, Touring Club de France. 
Cardot E., 1907b. La capitalisation forestière, Bulletin de la Société forestière des amis des arbres, cité dans Revue mensuelle du TCF, mars, 130.

Cardot E., 1911. L'impôt forestier, Revue mensuelle du TCF, février, 59-61.

Charlot M., 1901. Les arbres et l'école, Revue pédagogique, 5, 424430.

Coincy H. (de), 1905. Le premier congrès de l'aménagement des montagnes, Revue des Eaux et Forêts, 44, 577-588.

Comité de sites et monuments pittoresques, 1905. Séance du 14 novembre 1905, Revue mensuelle du TCF, décembre, 540.

Commission des pelouses et forêts du TCF, 1907. Forêts de protection, Revue mensuelle du TCF, novembre, 485-486.

Conseil d'administration du TCF, 1906. Compte rendu de la séance du 25 janvier 1906, Revue mensuelle du TCF, février, 85-87.

Conseil d'administration du TCF, 1913. Situation financière de l'association, exercice 1912-1913, Revue mensuelle du TCF, novembre, 490-491.

Corbin A. (Ed.), 1995. L'avènement des loisirs 1850-1960, Paris, Aubier.

Corbin A., 2001. L'Homme dans le paysage, Paris, Éditions Textuel.

Corvol A., 1987a. L'homme aux bois. Histoire des relations de l'homme et de la forêt (XV $V^{e}-X X^{e}$ siècles), Paris, Fayard.

Corvol A., 1987b. L'arbre et la nature (XVII $-\mathrm{XX}^{\mathrm{e}}$ siècles), Histoire, économie et société, 6, 1, 67-82.

Cousin S., Réau B., 2009. Sociologie du tourisme, Paris, La Découverte.

Defert H., 1906. Commission des pelouses et forêts, Revue mensuelle du TCF, décembre, 544-545.

Defert H., 1907a. Le cañon du Tarn, Revue mensuelle du TCF, mai 1907, 197.

Defert H., 1907b. Déboisements pyrénéens, Revue mensuelle du TCF, février, 54-56.

Defert H., 1907c. L'orientation des capitaux vers les placements forestiers, Revue mensuelle du TCF, décembre, 541-542.

Defert H., 1908. Dilapidations et reconstitutions forestières, Revue mensuelle du TCF, janvier, 8-10.

Defert H., 1909a. Conservation des forêts, Revue mensuelle du TCF, février, 62-65.

Defert H., 1909b. Enseignement sylvo-pastoral, Revue mensuelle du TCF, mars, 112.

Defert H., 1910. Syndicats forestiers, Revue mensuelle du TCF, juin, 246.

Defert H., 1911. L'impôt forestier, Revue mensuelle du TCF, mai, 200.

Detois E., 1906. La déforestation du plateau central, Revue mensuelle du TCF, août, 357-359.

Gauchon C., 2002. Les sites naturels classés entre 1906 et 1930 dans les Alpes du Nord : entre tourisme et protection, bilan et actualité, Revue de géographie alpine, 90, 2, 15-31.
Granet-Abisset A.-M., 2012. L'historien, les risques et l'environnement : un regard sur la nature et les hommes, in Thévenot D. (Ed.), Actes des 23e journées scientifiques de l'environnement - risques environnementaux: détecter, comprendre, s'adapter, 31 janvier-2 février 2012, Hôtel du département, Créteil, HAL, <hal-00675617>.

Kalaora B., Savoye A., 1986. La forêt pacifiée. Sylviculture et sociologie au XIXe siècle, Paris, L'Harmattan.

Larique B., 2006. L'économie du tourisme en France des années 1890 à la veille de la Seconde Guerre mondiale. Organisation et développement d'un secteur socio-économique. Thèse de doctorat en histoire, Université Bordeaux 3, Bordeaux.

Métailié J.-P., 2006. La "dégradation des montagnes» au XIX ${ }^{\mathrm{e}}$ siècle dans les Pyrénées, in Beck C., Luginbühl Y., Muxart T. (Eds), Temps et espaces des crises de l'environnement, Paris, Quæ, 191-210.

Morin G.-A., 2010. La continuité de la gestion des forêts françaises de l'Ancien Régime à nos jours, ou comment l'État a-t-il pris en compte le long terme, Revue française d'administration publique, 134, 233-248.

Peltier A., 2005. La gestion des risques naturels dans les montagnes d'Europe occidentale. Étude comparative du Valais (Suisse), de la Vallée d'Aoste (Italie) et des Hautes-Pyrénées (France). Thèse de doctorat en géographie, Université de Toulouse-Le Mirail, Toulouse.

Puyo J.-Y., 1999. La science forestière vue par les géographes français, ou la confrontation de deux sciences « diagonales » (1870-1914), Annales de Géographie, 108, 609-610, 615634.

Reclus O., 1905a. Le salut des sites par la protection des forêts, Revue mensuelle du TCF, juin, 248-250.

Reclus O., 1905b. L'association pour l'aménagement des montagnes, Revue mensuelle du TCF, octobre, 435-437.

Schut P.-O., 2013. Sport as a major player in the development of tourism: the history of mountaineering in the Pelvoux massif, France, from 1861 to 1914, International Journal of History of Sport, 30, 12, 1329-1350.

Schut P.-O., Levet-Labry E., 2014. Les relations entre tourisme et sport autour des Jeux olympiques de 1924, Revue STAPS, $105,37-49$.

Sénat, 1910. Compte rendu de la séance du 25 janvier 1910, JORF Débats Parlementaires, 26 janvier 1910.

Surell A., 1870 [1 $1^{\mathrm{re}}$ éd. : 1841]. Étude sur les torrents des HautesAlpes, Paris, Dunod.

Terral H., 2008. Le Touring Club de France et le ministère de l'Instruction publique: une coopération pédagogique au début du XX ${ }^{\mathrm{e}}$ siècle. In Actes du colloque international « L'éducation au développement durable. De l'école au campus », 25 au 27 juin 2008, Albi, http:/ / colloque-edd-2008.mines-albi.fr/ Donnees/archive_mercredi_am/31.pdf.

Terret T., 2010. Histoire du sport, Paris, PUF.

Reçu le 5 octobre 2015. Accepté le 6 octobre 2016. 